\title{
Ultrafast adiabatic manipulation of slow light in a photonic crystal
}

\author{
T. Kampfrath, ${ }^{1}$ D. M. Beggs, ${ }^{2}$ T. P. White, ${ }^{2}$ A. Melloni,${ }^{3}$ T. F. Krauss,${ }^{2}$ and L. Kuipers ${ }^{1}$ \\ ${ }^{1}$ Institute for Atomic and Molecular Physics (AMOLF), Science Park 104, NL-1098 XG Amsterdam, The Netherlands \\ ${ }^{2}$ School of Physics and Astronomy, University of St. Andrews, St. Andrews, Fife KY16 9SS, United Kingdom \\ ${ }^{3}$ Dipartimento di Elettronica e Informazione, Politecnico di Milano, Via Ponzio 34/5, I-20133 Milano, Italy
}

(Received 2 September 2009; published 27 April 2010)

\begin{abstract}
We demonstrate by experiment and theory that a light pulse propagating through a Si-based photonic-crystal waveguide is adiabatically blueshifted when the refractive index of the $\mathrm{Si}$ is reduced on a femtosecond time scale. Thanks to the use of slow-light modes, we are able to shift a 1.3-ps pulse at telecom frequencies by $0.3 \mathrm{THz}$ with an efficiency as high as $80 \%$ in a waveguide as short as $19 \mu \mathrm{m}$. An analytic theory reproduces the experimental data excellently, which shows that adiabatic dynamics are possible even on the femtosecond time scale as long as the external stimulus conserves the spatial symmetry of the system.
\end{abstract}

DOI: $10.1103 /$ PhysRevA.81.043837

PACS number(s): 42.65.Re, 42.70.Qs, 78.47.J-

\section{INTRODUCTION}

A physical system is said to evolve adiabatically when it remains in a stationary state of its instantaneous Hamiltonian. The only dynamics is then the modification of this eigenstate due to the Hamiltonian's temporal changes [1,2]. Such behavior often occurs in molecules where the electrons continuously adjust their orbitals to the current positions of the much slower moving nuclei [2]. While adiabatic processes are routinely used to efficiently and reversibly manipulate the quantum state of atoms [3], it was only recently that the adiabatic frequency shifting of light in a nanophotonic cavity was proposed [4] and demonstrated [5-8]. In these experiments, a fast external stimulus modified the cavity eigenfrequency and simultaneously the color of light residing inside the cavity. As this light represents only a fraction of the incident pulse, the conversion efficiency of cavities is intrinsically limited [9].

In order to host and, thus, shift an entire light pulse, the use of many coupled cavities $[9,10]$ or, more generally, photoniccrystal waveguides (PCWs) appears promising. A PCW can be tailored to support eigenstates (or eigenmodes) with a low group velocity over a terahertz bandwidth [11]. Using such slow light allows one to store complete picosecond light pulses in very short waveguides and, thus, to manipulate the light with little energy [12]. However, while a cavity supports only discrete eigenmodes, a PCW has a continuous mode spectrum. This difference makes adiabatic light dynamics in a PCW a nontrivial matter since, according to Born and Fock [1], a system evolves adiabatically "if a given perturbation is acting on it slowly enough and if there is a gap between the eigenvalue and the rest of the Hamiltonian's spectrum." So far, theoretical work on time-varying waveguides has mainly focused on ordinary waveguides [13], nonadiabatic aspects $[14,15]$, or numerical simulations [16].

In this paper, we make the step from a single cavity to a PCW and demonstrate the frequency conversion of a 1.3-ps pulse at telecom frequencies. Thanks to the use of slow light, the input pulse fits nearly entirely in the $19-\mu \mathrm{m}$ short actuated waveguide, and we obtain a frequency shift of $0.3 \mathrm{THz}$ with a power conversion efficiency of more than $80 \%$. An analytical theory based on the adiabatic approximation excellently reproduces the experimental data without using any fit parameters or mode calculations. The agreement implies that the observed frequency conversion proceeds adiabatically; that is, no energy exchange occurs among the eigenmodes of the waveguide. We identify the high spatial symmetry rather than the speed of the external stimulus as the crucial reason for this behavior.

\section{EXPERIMENT}

A schematic of the experiment is shown in Fig. 1. While a probe pulse propagates through a PCW, the instantaneous mode spectrum is shifted to higher frequencies by illuminating the PCW with an intense pump pulse. The waveguide is a so-called W1 PCW and can be pictured as a missing row of holes in a periodically perforated Si membrane (Fig. 1). Details on the fabrication and characterization can be found in Refs. [17,18]. The 220-nm thin waveguide is embedded in $\mathrm{SiO}_{2}$ and has a lattice periodicity $a$ of $390 \mathrm{~nm}$ and a length $L$ of only $48 a \approx 19 \mu \mathrm{m}$. We have calculated the eigenfrequency $\omega_{k} / 2 \pi$ of each waveguide mode with wave vector $k$ using a numerical three-dimensional plane-wave expansion [19], and the resulting dispersion relation is shown by the lower (red) curve in Fig. 2(a). Modes on the right-hand side of the $\mathrm{SiO}_{2}$ light line are bound to the PCW. The slope of $\omega_{k}$ at $k a / 2 \pi>$ 0.38 is so flat that the corresponding modes have a group velocity $v_{\mathrm{g}}=\partial_{k} \omega_{k}$ of $c / 30$ and even slower. Our calculations [19] also show that a change $\Delta n$ in the Si refractive index induces a frequency shift $\Delta \omega_{k}$ of each eigenmode $k$ which is proportional to $\Delta n$ and independent of $k$. For example, $\Delta n$
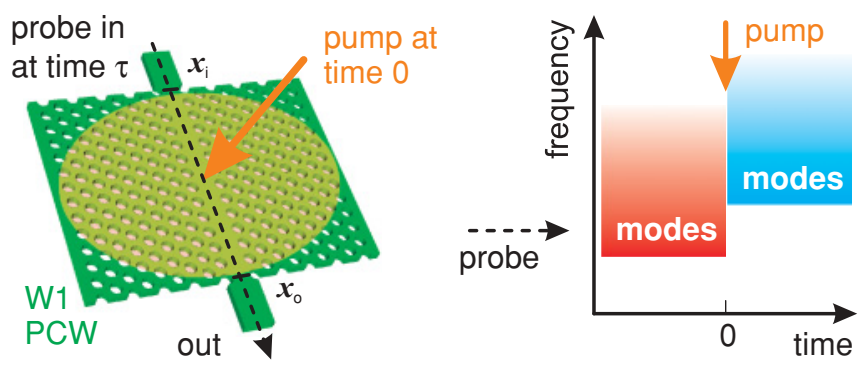

FIG. 1. (Color online) Experimental principle. While a probe pulse traverses a PCW, the absorption of a pump pulse leads to a sudden blueshift of the eigenmodes of the PCW. We detect the probe light at the PCW exit as a function of the delay $\tau$ between pump and probe pulse. 


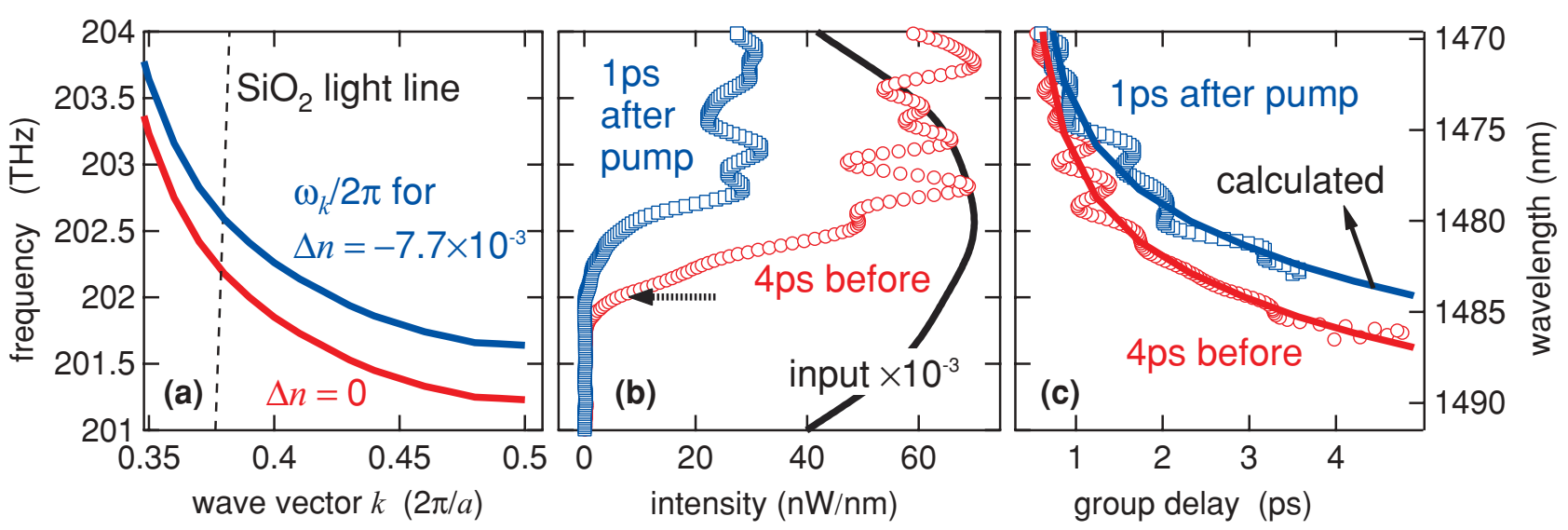

FIG. 2. (Color online) Characterization of the time-varying PCW. (a) Calculated dispersion relation $\omega_{k}$ for two changes $\Delta n$ in the Si refractive index. (b) Measured intensity spectrum $\left|E_{\mathrm{i}}(\omega)\right|^{2}$ of the broadband input probe pulse and the resulting output spectra $\left|E_{\mathrm{o}}(\omega, \tau)\right|^{2}$ at 4 ps before and 1 ps after arrival of the pump pulse. (c) Measured group delay of the output pulses of (b) together with curves $L / \partial_{k} \omega_{k}$ as calculated from (a).

of $-7.7 \times 10^{-3}$ leads to a global blueshift of $0.39 \mathrm{THz}$, as demonstrated by the upper (blue) curve in Fig. 2(a).

We realize the desired changes $\Delta n$ and, thus, $\Delta \omega_{k}$ on ultrashort time scales by means of a laser pulse which generates an electron-hole plasma in the Si parts of the PCW at time 0 (Fig. 1) [20,21]. The pump pulse has a duration of $100 \mathrm{fs}$ full width at half maximum of the intensity (FWHMI), a center wavelength of $810 \mathrm{~nm}$, and an energy of $2 \mathrm{~nJ}$. It is generated by a Ti:sapphire laser oscillator with an $80-\mathrm{MHz}$ pulse repetition rate. The resulting time-varying transmittance of the PCW is fully characterized over a large bandwidth using a Fourier-limited 180-fs, 1480-nm, 10-pJ probe pulse whose intensity spectrum $\left|E_{\mathrm{i}}(\omega)\right|^{2}$ is displayed by the black curve in Fig. 2(b). The probe pulse is provided by an optical parametric oscillator fed by the Ti:sapphire laser and enters the PCW at time $\tau$ set by an adjustable mechanical delay stage. At the PCW exit, the pulse is picked up by a lensed fiber, and we measure the complex-valued Fourier spectrum $E_{\mathrm{o}}(\omega, \tau)$ of its electric field by means of interference with a reference pulse in an optical spectrum analyzer [22]. We emphasize that the two-dimensional data set $E_{\mathrm{o}}(\omega, \tau)$ of the output field resulting from the broadband input $E_{\mathrm{i}}(\omega)$ is sufficient to extract the PCW output for any other input pulse [23].

\section{RESULTS}

\section{A. Broadband characterization}

We first consider the broadband output $E_{\mathrm{o}}(\omega, \tau)$ measured at delays of $\tau=-4$ and $1 \mathrm{ps}$, that is, for the probe pulse arriving long before and after the PCW has been modified by the pump pulse, respectively. While Fig. 2(b) shows the intensity spectra $\left|E_{\mathrm{o}}(\omega, \tau)\right|^{2}$, Fig. 2(c) displays the group delay $\partial_{\omega} \arg E_{\mathrm{o}}(\omega, \tau)$, which quantifies the transit time of a narrowband pulse with center frequency $\omega$ through the waveguide. Note that the group-delay spectrum of the pumped PCW is a blueshifted version of that of the unpumped PCW. Moreover, both delay curves exhibit a striking rise with decreasing frequency. These observations are consistent with the calculated dispersion relations of Fig. 2(a), where the blueshift and the slow-light modes with low group velocity $v_{\mathrm{g}}=\partial_{k} \omega_{k}$ are clearly visible. In fact, we can extract the group delay from the calculated $\omega_{k}$ using the expression $L / v_{\mathrm{g}}$. Excellent agreement with the measured data in Fig. 2(c) is found when we use the lower (red) and upper (blue) dispersion curves in Fig. 2(a) for the unpumped and pumped waveguide, respectively.

At group delays of $1 \mathrm{ps}$ and longer, the group velocity of less than $c / 15$ leads to increased light scattering at imperfections of the PCW. This scattering results in spectral modulations and a drop of the PCW output with decreasing frequency, as seen in Fig. 2(b) [24]. Absorption by transient free electrons and holes causes an additional reduction of the output of the pumped waveguide [leftmost (blue) curve in Fig. 2(b)]. We summarize that the eigenmodes of our PCW indeed undergo an ultrafast blueshift as anticipated in Fig. 1, and the frequency change amounts to $0.39 \mathrm{THz}$. As shown previously [21], the mode shift scales linearly with the pump power, but at the price of increased free-carrier absorption.

\section{B. Narrowband response: Blueshift}

We now investigate the effect of the time-varying eigenmodes on a picosecond input pulse propagating in the PCW. The resulting output is obtained from our measured broadband data set $E_{\mathrm{o}}(\omega, \tau)$ as follows [23]. Since the probe intensity is low, the output and input fields are in the time domain connected by the linear relationship

$$
E_{\mathrm{o}}(t, \tau)=\int d s \mathcal{T}(t, s) E_{\mathrm{i}}(s-\tau) .
$$

Here, the so-called transfer function $\mathcal{T}(t, s)$ fully characterizes the PCW response to the probe pulse. After $\mathcal{T}$ is inferred from the measured $E_{\mathrm{o}}(\omega, \tau)$ and $E_{\mathrm{i}}(\omega)$, we use Eq. (1) again to numerically extract the response of the PCW to any other input pulse that has a smaller bandwidth than the broadband input pulse shown in Fig. 2(b). Details on this procedure can be found in Ref. [23].

We apply this formalism to a Fourier-limited and 1.3-ps FWHMI long Gaussian input pulse centered at $\omega_{\mathrm{c}} / 2 \pi=$ $202 \mathrm{THz}$ as indicated by the arrow in Fig. 2(b). The major 


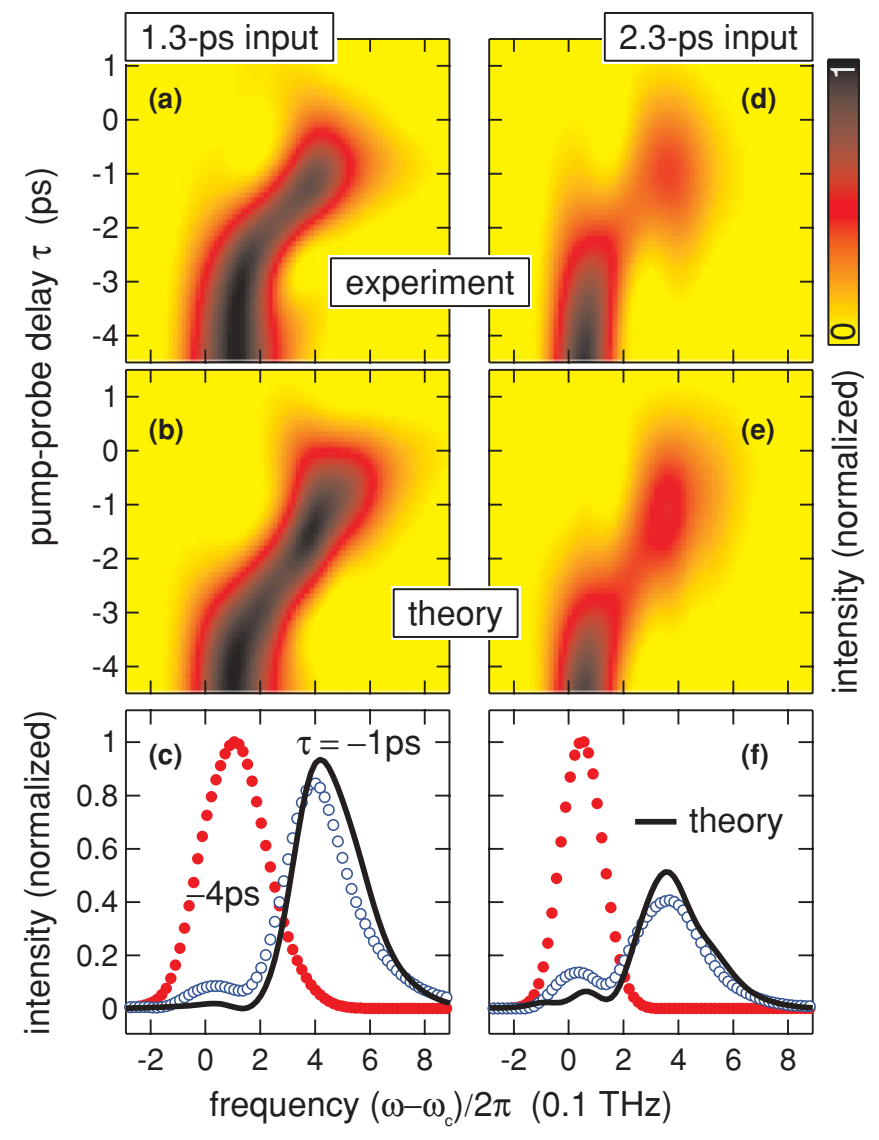

FIG. 3. (Color online) Ultrafast adiabatic frequency shifting. (a) Experimentally determined and (b) calculated intensity spectra of the PCW response to a 1.3-ps-long input pulse whose center frequency is indicated by the arrow in Fig. 2(b). (c) Measured and calculated output spectra at $\tau=-4$ ps and -1 ps. (d)-(f) Same as (a)-(c) but for a 2.3-ps input pulse.

part of this input pulse fits in the length of the PCW since its duration is shorter than its transit time of about $2.5 \mathrm{ps}$. Figure 3(a) shows the resulting output spectra as a function of the pump-probe delay. As expected from Fig. 2(b), there is maximum output before the arrival of the pump pulse at $\tau<-4$ ps, but a two-order-of-magnitude smaller output after the arrival at $\tau>1 \mathrm{ps}$. The spectral maximum of the output at $\tau<-4 \mathrm{ps}$ is shifted by about $0.1 \mathrm{THz}$ with respect to the center frequency of the input pulse. This shift is merely a filter effect and arises from the sharp drop of the PCW transmittance as seen in Fig. 2(b).

Note, however, the drastic effects around $\tau=-1$ ps when the pump and probe pulse have maximum overlap in the center of the PCW. A distinct new spectral peak at $\left(\omega-\omega_{\mathrm{c}}\right) / 2 \pi=$ $0.4 \mathrm{THz}$ appears and, as shown by the line cut in Fig. 3(c), it is a blueshifted version of the spectrum of the pulse passing the unpumped PCW. We emphasize that this peak cannot arise from a transiently increased transmittance at the new peak position because the throughput of the pumped system actually decreases at these frequencies [Fig. 2(b)].

Figure 3(c) demonstrates that our time-dependent PCW shifts the spectrum of the output pulse by about $0.3 \mathrm{THz}$, which agrees quite well with the mode shift of Fig. 2(a). Note that the blueshifted part of the output pulse contains an energy of more than $80 \%$ that of the pulse leaving the unpumped PCW. The values of shift and efficiency are comparable to those of a state-of-the-art $\mathrm{LiNbO}_{3}$ frequency shifter [25], but thanks to the use of slow light, our PCW is 200 times shorter. In comparison to nanophotonic cavities [5], the approach used here has a conversion efficiency more than one order of magnitude higher. This increase has two major reasons. First, our PCW is capable of hosting most of the 1.3-ps input pulse, which ensures maximum overlap between incident light and pumped $\mathrm{Si}$ volume. This assertion is corroborated by Figs. 3(d) and 3(f), which show the PCW output for a Fourierlimited input pulse with a longer duration of 2.3 ps FWHMI. In this case, only part of the input pulse fits in the actuated PCW, resulting in a significantly reduced conversion efficiency. Note that the spatial fraction of the pulse experiencing the mode shift is shorter than the total pulse, which explains the increased bandwidth of the frequency-shifted output [Fig. 3(f)]. Second, despite the use of slow light, the transit time of light through our PCW is still relatively short compared to that of cavities with storage times of more than 15 ps [5]. As a consequence, light absorption by transient free charge carriers is greatly reduced in our PCW.

\section{Theoretical description}

In order to understand the observed dynamics displayed in Figs. 3(a) and 3(d), we develop a simple yet rigorous analytical theory of the light propagation through a temporally changing PCW. Technical details of this model can be found in the Appendix. In brief, we calculate the transfer function $\mathcal{T}$ of the PCW by choosing an input pulse $\delta(t-\tau)$, which, according to Eq. (1), yields the desired $\mathcal{T}(t, \tau)$ as the output. The PCW response to any other input $E_{\mathrm{i}}(t)$ is then obtained by using Eq. (1).

The effects of the pump pulse and the photonic-crystal environment are contained in the time- and position-dependent dielectric function $\varepsilon^{t}(\boldsymbol{x})$. As a consequence, the orthonormalized eigenmodes $\boldsymbol{E}_{k}^{t}$ and eigenfrequencies $\omega_{k}^{t}$ of the PCW depend on time $t$ as well. We now make the crucial assumption of adiabatic dynamics; that is, we completely neglect scattering of light from one mode into another. As shown in the Appendix, the electric field of the probe pulse then evolves according to

$$
\boldsymbol{E}(\boldsymbol{x}, t)=\Theta(t-\tau) \sum A_{k} \exp \left(-\mathrm{i} \int_{\tau}^{t} d s \omega_{k}^{s}\right) \boldsymbol{E}_{k}^{t}(\boldsymbol{x}),
$$

where $\Theta$ denotes the Heaviside step function, and $A_{k}$ is $\varepsilon^{\tau}\left(\boldsymbol{x}_{\mathrm{i}}\right) \boldsymbol{E}_{k}^{\tau *}\left(\boldsymbol{x}_{\mathrm{i}}\right) \cdot \boldsymbol{e}_{\mathrm{i}}$ with $\boldsymbol{e}_{\mathrm{i}}$ being the polarization of the input pulse. Equation (2) is the central result of this section and has a quite intuitive interpretation: When the laser generates the $\delta$-like probe pulse at time $\tau$, the amplitude of each mode $k$ jumps from zero to $A_{k}$, resulting in the initial field $\boldsymbol{E}(\boldsymbol{x}, \tau)=\boldsymbol{e}_{\mathrm{i}} \delta\left(\boldsymbol{x}-\boldsymbol{x}_{\mathrm{i}}\right)$. Subsequently, the mode amplitudes evolve independently of each other and accumulate the typical phase shift $\int_{\tau}^{t} d s \omega_{k}^{s}$ in the course of time.

The actual measured output signal is the projection of the probe field at the detector position on the output polarization. Note that, in our PCW, the pump pulse shifts all eigenfrequencies $\omega_{k}$ by the same frequency $\Delta \omega^{t}=\omega_{k}^{t}-\omega_{k}$ (Fig. 2), which 
drastically simplifies Eq. (2). As shown in the Appendix, the resulting transfer function

$$
\mathcal{T}(t, \tau)=\mathcal{T}_{0}(t, \tau) \exp \left(-\mathrm{i} \int_{\tau}^{t} d s \Delta \omega^{s}\right)
$$

is just the transfer function $\mathcal{T}_{0}$ of the unpumped PCW multiplied by a factor which contains the additionally acquired phase due to the pump-induced detuning of the eigenfrequencies. We emphasize that knowledge of the eigenfrequencies and eigenmodes of the PCW is not necessary to calculate $\mathcal{T}$. The required $\mathcal{T}_{0}(t, \tau)=\mathcal{T}_{0}(t-\tau, 0)$ is obtained from our experimental data of Fig. 2 for $\tau=-4$ ps, where $\mathcal{T}(t, \tau)=\mathcal{T}_{0}(t, \tau)$ holds. Furthermore, we assume a steplike frequency shift $\Delta \omega^{t}=\Delta \omega \Theta(t)$, where $\Delta \omega / 2 \pi=0.39 \mathrm{THz}$ has been obtained from Fig. 2(c) as discussed above.

Figure 3 shows the results of our calculations based on Eqs. (1) and (3). The modeling reproduces amplitude (Fig. 3) and phase (not shown) of our experimental spectra excellently, both for the short and long input pulse, for all pump-probe delays $\tau$, without any fit parameters. For the particular case when the input pulse resides completely in the PCW during pump excitation, inspection of Eqs. (1) and (3) shows that the output of the pumped PCW equals precisely that of the unpumped PCW shifted by the frequency $\Delta \omega$. However, both measured and modeled spectra of Fig. 3(c) exhibit a frequency shift of approximately $0.3 \mathrm{THz}$, which is smaller than the 0.39-THz mode shift as inferred from Fig. 2. These facts show that the 1.3-ps probe pulse still does not fit entirely in the PCW. We finally note that losses due to free-carrier absorption are not accounted for in our theory, which explains why the modeled output pulse has more energy than the one measured [Fig. 3(c)].

\section{Discussion}

The striking agreement of measured and calculated output spectra justifies the assumption of adiabatic dynamics. This result is surprising because the usual derivation of the adiabatic theorem [1,2] relies on a change of the "potential energy" $\varepsilon^{t}$ that is much slower than the inverse eigenfrequency spacing $1 /\left(\omega_{k}^{t}-\omega_{k^{\prime}}^{t}\right)$. For our waveguide with its continuous mode spectrum, this condition is certainly violated, and the $100-\mathrm{fs}$ fast change in $\varepsilon^{t}$ could easily induce scattering of the probe light between modes $k$ and $k^{\prime}$ that are up to several terahertz apart. Indeed, such sideband generation was recently reported for a nanocavity [26]. On the other hand, as shown in the Appendix, no transfer of light between modes with different wave vector occurs, provided the pump-pulse illumination respects the discrete translational symmetry of the PCW. Along these lines, coupling of modes with equal wave vector from adjacent dispersion bands is excluded also as these modes exhibit different symmetry with respect to spatial reflections. Therefore, the dominance of adiabatic effects in the propagation of light through a homogeneously actuated $\mathrm{PCW}$ is a consequence of symmetry conservation.

\section{CONCLUSION}

The frequency conversion of light reported here highlights the high efficiency of adiabatic processes provided the light pulse is fully contained in the actuated volume. Our approach relies on slow-light modes $k$ which all undergo the same frequency shift. A wealth of further exciting possibilities to manipulate light in a PCW can be expected if the shift $\Delta \omega_{k}$ exhibits a strong $k$ dependence. As suggested previously [16], a pump-induced flattening of the dispersion curve $\omega_{k}$ could be used to adiabatically slow down light as it propagates through a photonic structure. We emphasize that slow light lends itself ideally to this application: Compared to fast light, the low group velocity $v_{\mathrm{g}}$ of slow light will undergo much larger relative changes $\Delta v_{\mathrm{g}} / v_{\mathrm{g}}$, where $\Delta v_{\mathrm{g}}=\partial_{k} \Delta \omega_{k}$ is the pump-induced modification of the group velocity. The theory of light propagation through time-varying PCWs presented here may help explore and optimize such adiabatic manipulation schemes of light in nanophotonic structures.

\section{ACKNOWLEDGMENTS}

We thank K. Campen, F. Koenderink, and A. Tip for discussions and acknowledge funding through the European Union FP6-FET "SPLASH" project. This work is part of the research program of the Foundation for Fundamental Research on Matter (FOM), which is financially supported by the Netherlands Organisation for Scientific Research (NWO).

\section{APPENDIX: MODEL DETAILS}

\section{A. Adiabatic transfer function}

Figure 4 shows a model schematic of our experiment. A pointlike probe laser with linear polarization $\boldsymbol{e}_{\mathrm{i}}$ and a detector are placed next to the input and output facet of a PCW at positions $\boldsymbol{x}=\boldsymbol{x}_{\mathrm{i}}$ and $\boldsymbol{x}_{\mathrm{o}}$, respectively. To avoid the tedious treatment of possible light reflections at the facets, we fictitiously extend the waveguide infinitely. We calculate the transfer function of the PCW by choosing an input pulse $E_{\mathrm{i}}(t-\tau)=\delta(t-\tau)$, which, according to Eq. (1), yields the desired $E_{\mathrm{o}}(t, \tau)=\mathcal{T}(t, \tau)$ as the output. Here, $t$ denotes time and $\tau$ the arrival time of the input pulse at the PCW entrance. The transfer function $\mathcal{T}$ contains all information relevant to the transmittance of our waveguide, for example, the PCW length and dispersion. Once $\mathcal{T}$ is known, we can use Eq. (1) to calculate the PCW response to any other input $E_{\mathrm{i}}(t)$, for instance, pulses with different duration and chirp.

Since we consider a $\delta$-like input pulse, the dynamics of the probe pulse for times $t>\tau$ is determined by the sourcefree Maxwell equations, which are written in the form of the Schrödinger equation [27]

$$
\mathrm{i} \partial_{t} \psi=\mathcal{H}^{t} \boldsymbol{\psi}
$$

Here, the state

$$
\boldsymbol{\psi}=\left(\begin{array}{c}
\varepsilon^{t} \boldsymbol{E} \\
\boldsymbol{B}
\end{array}\right)
$$

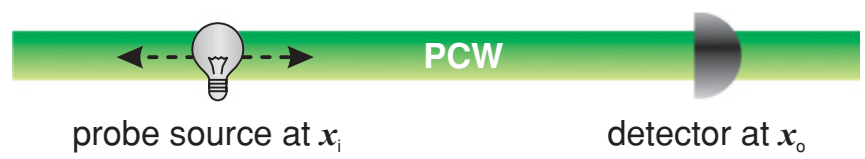

FIG. 4. (Color online) Model schematic of the experimental situation. The pointlike probe laser with linear polarization $\boldsymbol{e}_{\mathrm{i}}$ and a detector are placed next to the input and output facet of the waveguide at positions $\boldsymbol{x}=\boldsymbol{x}_{\mathrm{i}}$ and $\boldsymbol{x}_{\mathrm{o}}$. 
of the light field contains the electric field $\boldsymbol{E}(\boldsymbol{x}, t)$, the magnetic field $\boldsymbol{B}(\boldsymbol{x}, t)$, and the time- and position-dependent dielectric function $\varepsilon^{t}(\boldsymbol{x})$. The operator

$$
\mathcal{H}^{t}=\frac{c}{\mathrm{i}}\left(\begin{array}{cc}
0 & -\nabla \times \\
\nabla \times\left(. / \varepsilon^{t}\right) & 0
\end{array}\right)
$$

governs the dynamics of $\psi$. It is Hermitian with respect to the scalar product

$$
\left\langle\boldsymbol{\psi}_{1}, \boldsymbol{\psi}_{2}\right\rangle=\int d^{3} \boldsymbol{x}\left(\varepsilon^{t} \boldsymbol{E}_{1}^{*} \cdot \boldsymbol{E}_{2}+\boldsymbol{B}_{1}^{*} \cdot \boldsymbol{B}_{2}\right) .
$$

Therefore, $\mathcal{H}^{t}$ possesses a system of eigenfrequencies $\omega_{k}^{t}$ and eigenmodes $\boldsymbol{\psi}_{k}^{t}=\left(\varepsilon^{t} \boldsymbol{E}_{k}^{t}, \boldsymbol{B}_{k}^{t}\right)$ which can be chosen to be orthonormalized, $\left\langle\boldsymbol{\psi}_{k}^{t}, \boldsymbol{\psi}_{k^{\prime}}^{t}\right\rangle=2 \delta_{k k^{\prime}}$. Due to the influence of the pump pulse, the dielectric function $\varepsilon^{t}(\boldsymbol{x})$ is generally time-dependent, and so are the scalar product, the operator $\mathcal{H}^{t}$, and its eigenmodes $\psi_{k}^{t}$ and eigenfrequencies $\omega_{k}^{t}$ [27]. We now expand the light field with respect to the instantaneous eigenmodes,

$$
\boldsymbol{\psi}(\boldsymbol{x}, t)=\sum a_{k}(t) \boldsymbol{\psi}_{k}^{t}(\boldsymbol{x})
$$

Inserting Eq. (A5) in Eq. (A1) yields the equation of motion for the mode amplitudes $a_{k}(t)$,

$$
\partial_{t} a_{k}=-\mathrm{i} \omega_{k}^{t} a_{k}-\mathrm{i} \sum_{k^{\prime}} \Gamma_{k k^{\prime}}^{t} a_{k^{\prime}},
$$

where the rate $\Gamma_{k k^{\prime}}^{t}$ of amplitude transfer from mode $k$ to $k^{\prime}$ is given by the overlap integral

$$
2 \mathrm{i} \Gamma_{k k^{\prime}}^{t}=\left\langle\boldsymbol{\psi}_{k}^{t}, \partial_{t} \boldsymbol{\psi}_{k^{\prime}}^{t}\right\rangle .
$$

Adiabatic behavior arises when the coupling between different modes $k$ and $k^{\prime}$ can be neglected; that is, $\Gamma_{k k^{\prime}}^{t}=0$. Then, Eq. (A6) results in the adiabatic solution

$$
a_{k}(t)=A_{k} \Theta(t-\tau) \exp \left(-\mathrm{i} \int_{\tau}^{t} d s \omega_{k}^{s}\right),
$$

where the Heaviside step function $\Theta$ comes into play through the boundary condition $\boldsymbol{\psi}(\boldsymbol{x}, t)=0$ if $t<\tau$. The initial mode amplitude

$$
A_{k}=\left\langle\boldsymbol{\psi}_{k}^{t}, \boldsymbol{\psi}(., \tau)\right\rangle / 2=\varepsilon^{\tau}\left(\boldsymbol{x}_{\mathrm{i}}\right) \boldsymbol{E}_{k}^{\tau *}\left(\boldsymbol{x}_{\mathrm{i}}\right) \cdot \boldsymbol{e}_{\mathrm{i}}
$$

follows from the boundary condition $\boldsymbol{E}(\boldsymbol{x}, \tau)=\boldsymbol{e}_{\mathrm{i}} \delta\left(\boldsymbol{x}-\boldsymbol{x}_{\mathrm{i}}\right)$. Putting Eqs. (A5), (A8), and (A9) together finally yields the desired Eq. (2) of the main text,

$$
\boldsymbol{E}(\boldsymbol{x}, t)=\Theta(t-\tau) \sum A_{k} \exp \left(-\mathrm{i} \int_{\tau}^{t} d s \omega_{k}^{s}\right) \boldsymbol{E}_{k}^{t}(\boldsymbol{x}) .
$$

Note that if the time-conjugate modes $\boldsymbol{\psi}_{k}^{t *}$ with negative eigenfrequencies $-\omega_{k}^{t}$ are ignored, $\boldsymbol{E}(\boldsymbol{x}, t)$ is twice the real part of the right-hand side of Eq. (A10).

As seen in Fig. 4, the actual measured output signal is the projection of the probe field at the detector position $\boldsymbol{x}_{\mathrm{o}}$ onto the output polarization $\boldsymbol{e}_{\mathrm{o}}$; that is, $\mathcal{T}(t, \tau)=\boldsymbol{E}\left(\boldsymbol{x}_{\mathrm{o}}, t\right) \cdot \boldsymbol{e}_{\mathrm{o}}$. For the PCW used in our experiment, all eigenfrequencies $\omega_{k}$ of the unpumped system are shifted by the same value $\Delta \omega^{t}=$ $\omega_{k}^{t}-\omega_{k}$, where $\omega_{k}$ is the frequency of eigenmode $\psi_{k}$ of the unpumped PCW. When we assume a negligible pump-induced modification of the eigenmodes, $\boldsymbol{\psi}_{k}^{t}=\boldsymbol{\psi}_{k}$, Eq. (A10) turns into the surprisingly simple Eq. (3) of the main text,

$$
\mathcal{T}(t, \tau)=\mathcal{T}_{0}(t, \tau) \exp \left(-\mathrm{i} \int_{\tau}^{t} d s \Delta \omega^{s}\right) .
$$

Note that Eq. (A11) is valid at positive frequencies $\omega$ of the Fourier-transformed $\mathcal{T}(\omega, \tau)=\int d t \exp (\mathrm{i} \omega t) \mathcal{T}(t, \tau)$. Negative frequencies are covered by using the relation $\mathcal{T}(-\omega, \tau)=$ $\mathcal{T}(\omega, \tau)^{*}$.

\section{B. Symmetry considerations}

We finally consider the mode-coupling rates $\Gamma_{k k^{\prime}}^{t}$ of Eq. (A7) in terms of the spatial periodicity of the PCW. As the actuating pump pulse respects all spatial symmetries of the PCW, the eigenmodes, $\boldsymbol{\psi}_{k}^{t}$ of $\mathcal{H}^{t}$ are always Bloch modes with $\mathcal{S}_{n a} \boldsymbol{\psi}_{k}^{t}=\exp (\mathrm{i} k n a) \boldsymbol{\psi}_{k}^{t}$. Here, $\mathcal{S}_{n a}$ denotes the translation by an integer multiple $n a$ of the lattice constant $a$ along the PCW direction. Therefore, one has $\left\langle\boldsymbol{\psi}_{k}^{t}, \mathcal{S}_{-n a} \mathcal{S}_{n a} \partial_{t} \boldsymbol{\psi}_{k^{\prime}}^{t}\right\rangle=$ $\left\langle\mathcal{S}_{n a} \boldsymbol{\psi}_{k}^{t}, \partial_{t} \mathcal{S}_{n a} \boldsymbol{\psi}_{k^{\prime}}^{t}\right\rangle$, which implies

$$
\Gamma_{k k^{\prime}}^{t}=\Gamma_{k k^{\prime}}^{t} \exp \left[\mathrm{i}\left(k^{\prime}-k\right) n a\right] .
$$

As a consequence, we have $\Gamma_{k k^{\prime}}^{t}=0$ if $k \neq k^{\prime}$. In other words, the pump pulse does not couple modes with different wave vectors [14]. A similar argument applies to modes having equal wave vectors yet odd and even symmetry with respect to spatial reflections.
[1] M. Born and V. A. Fock, Z. Phys. 51, 165 (1928).

[2] A. Messiah, Quantum Mechanics (Dover Publications, 1999).

[3] K. Bergmann, H. Theuer, and B. W. Shore, Rev. Mod. Phys. 70, 1003 (1998).

[4] M. Notomi and S. Mitsugi, Phys. Rev. A 73, 051803(R) (2006).

[5] S. F. Preble, Q. Xu, and M. Lipson, Nature Photon. 1, 293 (2007).

[6] T. Tanabe, M. Notomi, H. Taniyama, and E. Kuramochi, Phys. Rev. Lett. 102, 043907 (2009).

[7] Q. Lin, T. J. Johnson, C. P. Michael, and O. Painter, Opt. Express 16, 14801 (2008).

[8] A. M. Yacomotti et al., Phys. Rev. Lett. 96, 093901 (2006).
[9] Z. Gaburro et al., Opt. Express 14, 7270 (2006).

[10] F. Morichetti, C. Ferrari, and A. Melloni, in Proceedings of the 9th International Conference on Transparent Optical Networks (IEEE, New York, 2007), Vol. 3, pp. 75-78.

[11] T. Baba, Nature Photon. 2, 465 (2008).

[12] M. Soljačić and J. D. Joannopoulos, Nat. Mater. 3, 211 (2004).

[13] B. Felsen and G. M. Whitman, IEEE Trans. Antennas Propag. 18, 242 (1970).

[14] J. N. Winn, S. Fan, J. D. Joannopoulos, and E. P. Ippen, Phys. Rev. B 59, 1551 (1999).

[15] Z. Yu and S. Fan, Nature Photon. 3, 91 (2009).

[16] M. F. Yanik and S. Fan, Phys. Rev. Lett. 92, 083901 (2004). 
[17] D. M. Beggs, L. O'Faolain, and T. F. Krauss, Photonics Nanostruct. 6, 213 (2008).

[18] T. P. White, L. O'Faolain, Juntao Li, L. C. Andreani, and T. F. Krauss, Opt. Express 16, 17076 (2008).

[19] We used the freely available MIT Photonic Bands package; see S. G. Johnson and J. D. Joannopoulos, Opt. Express 8, 173 (2001).

[20] H. W. Tan, H. M. van Driel, S. L. Schweizer, and R. B. Wehrspohn, Phys. Rev. B 72, 165115 (2005).

[21] T. Kampfrath et al., Appl. Phys. Lett. 94, 241119 (2009).
[22] L. Lepetit, G. Chériaux, and M. Joffre, J. Opt. Soc. Am. B 12, 2467 (1995).

[23] T. Kampfrath, D. M. Beggs, T. F. Krauss, and L. Kuipers, Opt. Lett. 34, 3418 (2009).

[24] N. Le Thomas et al., Phys. Rev. B 80, 125332 (2009).

[25] D. A. Farias and J. N. Eckstein, IEEE J. Quantum Electron. 41, 94 (2005).

[26] P. Dong, S. F. Preble, J. T. Robinson, S. Manipatruni, and M. Lipson, Phys. Rev. Lett. 100, 033904 (2008).

[27] S. G. Johnson, http://math.mit.edu/stevenj/18.369/waveequations.pdf. 